\title{
Cyclic Flats of a Polymatroid
}

\author{
Dedicated to the memory of Frantisek Matúš
}

\author{
Laszlo Csirmaz@
}

\begin{abstract}
Polymatroids can be considered as "fractional matroids" where the rank function is not required to be integer valued. Many, but not every notion in matroid terminology translates naturally to polymatroids. Defining cyclic flats of a polymatroid carefully, the characterization by Bonin and de Mier of the ranked lattice of cyclic flats carries over to polymatroids. The main tool, which might be of independent interest, is a convolution-like method which creates a polymatroid from a ranked lattice and a discrete measure. Examples show the ease of using the convolution technique.
\end{abstract}

Mathematics Subject Classification. 03G10, 05B35.

Keywords. Polymatroid, Cyclic flats, Convolution, Ranked lattice.

\section{Introduction}

Cyclic flats of a matroid played an important role in matroid theory. They form a ranked lattice, i.e., a lattice with a non-negative number assigned to lattice elements. Bonin and de Mier in [1] gave a characterization of the ranked lattices arising this way. They quote Sims [11], where the rank function of the embedding matroid is specified explicitly by some convolution-like formula.

Generalizing cyclic flats to polymatroids is not completely straightforward, see [5] and [12] where the same definition of polymatroidal cyclic flats arose as we use in this paper. Our main contribution is a complete characterization of the ranked lattice of cyclic flats of a polymatroid together with singleton ranks. Motivated by Sims' construction [11], the convolution of a ranked lattice and a discrete measure (determined by the singleton ranks) is defined. This definition is an extension of the usual convolution of polymatroids, for details see [9]. Similarly to the matroid case, this convolution recovers the embedding polymatroid for the given ranked lattice and measure. We carefully identify the role of different conditions. Knowing which condition ensures what property 
allows us to use the convolution to create polymatroids with desired properties. It is illustrated by a simple example. A more substantial application is in $[3]$.

Traditionally, convolution is symmetric. In the last section we propose the convolution of two ranked lattices which is symmetric and falls back to the previous notion when the second lattice is a complete modular polymatroid. In a special case this convolution gives an interesting polymatroid extension. It is open under which general and useful conditions will the convolution be a polymatroid.

\section{Preliminaries}

\subsection{Notation}

All sets in this paper are finite. A polymatroid $\mathcal{M}=(f, M)$ is a non-negative, monotone and submodular function $f$ defined on the subsets of $M$. Here $M$ is the ground set, and $f$ is the rank function. A polymatroid is integer if the rank function takes integer values only, and it is a matroid if it is integer, and all singletons have value either zero or one. For a thorough treatment of matroids see [10]. Polymatroids were introduced by Edmonds [4], relevant results on polymatroids can be found, e.g., in $[2,7,8]$.

A polymatroid can be considered as "fractional matroid". Relaxing a combinatorial notion to its fractional version allows different techniques to apply. This not different in case of polymatroids. While tools handling matroids are mainly combinatorial, polymatroids have a nice geometrical interpretation allowing geometrical (and continuity) reasoning.

Following the usual practice, ground sets and their subsets are denoted by capital letters, their elements by lower case letters. The union sign is frequently omitted as well as the curly brackets around singletons, thus $a b A$ denotes the set $\{a, b\} \cup A$. The set difference has lower priority than union or intersection, thus $a A-b \cap B$ denotes $(\{a\} \cup A)-(\{b\} \cap B)$. For a function $f$ defined on subsets of a set the usual abbreviations are used. For a singleton $i \in C$ we write $f(i \mid C-i)$ for $f(C)-f(C-i)$.

A (discrete) measure $\mu$ on $M$ is an additive function on the subsets of $M$ with $\mu(\emptyset)=0$. As $M$ is finite, the measure is determined by its value on the singletons as

$$
\mu(A)=\sum\{\mu(a): a \in A\} .
$$

If $\mathcal{M}=(f, M)$ is a polymatroid, then the measure $\mu_{\mathcal{M}}$, or just $\mu_{f}$, is the one defined by the singleton ranks. Submodularity implies $\mu_{f}(A) \geq f(A)$ for all $A \subseteq M$.

A collection of subsets is a lattice $\mathcal{L}$ if any two elements $Z_{1}, Z_{2} \in \mathcal{L}$ have, in $\mathcal{L}$, a least upper bound - their join -, and a greatest lower bound - their meet -, using the standard subset relation as the ordering. The notation for join and meet are $Z_{1} \vee Z_{2}$ and $Z_{1} \wedge Z_{2}$, respectively. We write $Z_{1}<Z_{2}$ if $Z_{1}$ is strictly below $Z_{2}$, which is the same as $Z_{1} \subset Z_{2}$. The lattice elements $Z_{1}$ and $Z_{2}$ are incomparable if they are different and neither $Z_{1}<Z_{2}$ nor $Z_{2}<Z_{1}$ 
holds. As $\mathcal{L}$ is finite, it has a smallest element (the meet of all $Z \in \mathcal{L}$ ), denoted by $O_{\mathcal{L}}$, and a largest element (the join of all $Z \in \mathcal{L}$ ), denoted by $I_{\mathcal{L}}$. The pair $(\lambda, \mathcal{L})$ is a ranked lattice if the rank function $\lambda$ assigns non-negative real values to lattice elements. $\lambda$ is pointed if $\lambda\left(O_{\mathcal{L}}\right)=0$, and monotone if $Z_{1} \leq Z_{2}$ implies $\lambda\left(Z_{1}\right) \leq \lambda\left(Z_{2}\right)$.

\subsection{Flats, Cyclic Flats}

In this section $\mathcal{M}=(f, M)$ is a fixed polymatroid. The element $a \in M$ is a loop if it has rank zero: $f(a)=0$, in which case $f(a A)=f(A)$ for all $A \subseteq M$. The element $a \in M$ is a coloop or isthmus if $f(a \mid M-a)=f(a)$, which means $f(a A)=f(A)+f(a)$ for every $A \subset M$ not containing $a$. Both loops and coloops add trivial structural properties only, thus very frequently the polymatroid is, or can be, assumed to have no loops and no coloops.

The subset $F \subseteq M$ is a flat if proper extensions of $F$ have strictly larger ranks. The intersection of flats is a flat, and the closure of $A \subseteq M$, denoted by $\mathrm{cl}_{\mathcal{M}}(A)$, or simply by $\mathrm{cl}(A)$ when $\mathcal{M}$ is clear from the context, is the smallest flat containing $A$. The ground set $M$ is always a flat. Flats of a polymatroid form a lattice where the meet is the intersection, and the join is the closure of the union. The minimal flat is the collection of all loops, which is the empty set if $\mathcal{M}$ has no loops.

The flat $C \subseteq M$ is cyclic if for all $i \in C$ either $i$ is a loop, or $f(i \mid C-i)<$ $f(i)$, see $[5,12]$. In particular, the minimal flat (containing only loops) is cyclic. When $\mathcal{M}$ is a matroid, this definition of cyclic flats is equivalent to the original one, namely that $C$ is the union of circuits (minimal connected sets), see $[1,10]$.

Similar to the matroid case, cyclic flats form a lattice. The proof relies on the following structural property of cyclic flats, which appeared in $[3$, Section $3]$

Lemma 1. Every flat $F$ contains a unique maximal cyclic flat $C \subseteq F$; moreover for all $C \subseteq A \subseteq F$ we have

$$
f(A)=f(C)+\mu_{f}(A-C) .
$$

Proof. First, we show that $F$ contains a maximal cyclic flat $C$ with the given property, then we show that the maximal cyclic flat inside $F$ is unique.

Start with $F_{1}=F$, and suppose we have defined $F_{j}$ for some $j \geq 1$. If there is an element $x_{j} \in F_{j}$ such that $f\left(x_{j}\right)>0$ and $f\left(F_{j}\right)-f\left(F_{j}-x_{j}\right)=f\left(x_{j}\right)$, then let $F_{j+1}=F_{j}-x_{j}$, otherwise stop. Submodularity gives that for each $i<j$ $f\left(x_{i} F_{j}\right)=f\left(x_{i}\right)+f\left(F_{j}\right)>f\left(F_{j}\right)$, thus $F_{j}$ is a flat (as $F_{j}$ has smaller rank than $x_{i} F_{j}$ for every $x_{i} \in F-F_{j}$ ), and the last $F_{j}=C$ is cyclic. As no proper extension of $C$ inside $F$ is cyclic, it is a maximal cyclic flat in $F$ with the claimed property.

Second, suppose $C \subseteq F_{1}=F$ is a maximal cyclic flat. If $i \in C$ is not a loop then $f\left(i \mid F_{1}-i\right) \leq f(i \mid C-i)<f(i)$, consequently $C$ is a subset of

$$
F_{2}=\left\{i \in F_{1}: i \text { is a loop, or } f\left(i \mid F_{1}-i\right)<f(i)\right\} .
$$

Define similarly the sets $F_{1} \supseteq F_{2} \supseteq F_{3} \supseteq \cdots \supseteq C$. It is clear that each $F_{j}$ is a flat, and when $F_{j}=F_{j+1}$ then it is cyclic. As it contains $C$, it must equal $C$. 
Corollary 2. The lattice of cyclic flags, together with the rank of each cyclic flat and the rank of each singleton determine the polymatroid.

Proof. This is so as the rank of $A$ is the minimum of $f(C)+\mu_{f}(A-C)$ as $C$ runs over all cyclic flats. Indeed, $f(A) \leq f(C)+f(A-C) \leq f(C)+\mu_{f}(A-C)$ by submodularity, thus it is enough to show that for some cyclic flat $C$ equality holds. Let $F=\mathrm{cl}(A)$ and $C \subseteq F$ be the maximal cyclic flat in $F$. Then $f(F)=f(A)=f(A C)$, and by Lemma 1 ,

$$
f(A C)=f(C)+\mu_{f}(A C-C)=f(C)+\mu_{f}(A-C),
$$

as required.

Claim 3. Cyclic flats of a polymatroid form a lattice.

Proof. Let $C_{1}$ and $C_{2}$ be cyclic flats. Then $C_{1} \cap C_{2}$ is a flat which contains a unique maximal cyclic flat by Lemma 1 above. This is the largest cyclic flat below $C_{1}$ and $C_{2}$. The smallest upper bound of $C_{1}$ and $C_{2}$ is $C=\operatorname{cl}\left(C_{1} \cup C_{2}\right)$. Indeed, this is a flat, and we claim that it is also cyclic. If $i \in C-C_{1} C_{2}$ then $i$ is not a loop (as loops are in $C_{1} \cap C_{2}$ ), $f\left(i C_{1} C_{2}\right)=f(C)$, thus $f(i \mid C-i)=0<$ $f(i)$. If, say, $i \in C_{1}$ and $f(i)>0$ then $f(i \mid C-i) \leq f\left(i \mid C_{1}-i\right)<f(i)$ proving that $C$ is cyclic indeed.

\subsection{Convolution}

Let $(\lambda, \mathcal{L})$ be a ranked lattice and $\mu$ be a (discrete) measure both defined on subsets of $M$. The convolution of the ranked lattice and the measure, denoted by $\lambda * \mu$, assigns a non-negative value to each subset of $M$ as follows:

$$
\lambda * \mu: A \mapsto \min \{\lambda(Z)+\mu(A-Z): Z \in \mathcal{L}\} .
$$

In subsequent sections we write $r$ instead of $\lambda * \mu$. When $\mathcal{L}$ contains all subsets of $M$ and $\lambda$ is the rank function of a polymatroid, then (1) is equivalent to the usual convolution of two polymatroids, see $[7,9]$.

\section{Characterizing cyclic flats}

\subsection{Conditions}

Convolution will be used to recover a polymatroid from the lattice of its cyclic flats. Different conditions on the ranked lattice and the measure ensure different properties of the convolution. Rather than listing them repeatedly, we specify them here. In what follows, $(\lambda, \mathcal{L})$ is a ranked lattice and $\mu$ is a measure, both defined on subsets of the same set.

(Z1) $\lambda\left(O_{\mathcal{L}}\right)=0$, i.e., $\lambda$ is pointed.

(Z2) for comparable lattice elements $Z_{1} \leq Z_{2} \in \mathcal{L}$,

$$
0 \leq \lambda\left(Z_{2}\right)-\lambda\left(Z_{1}\right) \leq \mu\left(Z_{2}-Z_{1}\right) .
$$

(Z3) for different comparable lattice elements $Z_{1}<Z_{2} \in \mathcal{L}$,

$$
0<\lambda\left(Z_{2}\right)-\lambda\left(Z_{1}\right)<\mu\left(Z_{2}-Z_{1}\right) .
$$


$\left(\mathrm{Z}^{*}\right)$ for any two lattice elements $Z_{1}, Z_{2} \in \mathcal{L}$,

$$
\lambda\left(Z_{1}\right)+\lambda\left(Z_{2}\right) \geq \lambda\left(Z_{1} \vee Z_{2}\right)+\lambda\left(Z_{1} \wedge Z_{2}\right)+\mu\left(Z_{1} \cap Z_{2}-Z_{1} \wedge Z_{2}\right) .
$$

(Z3) if $a \in Z \in \mathcal{L}$, then $\mu(a) \leq \lambda(Z)$.

(Z4) (a) $\lambda(Z)>0$ for $Z \neq O_{\mathcal{L}}$; (b) $\mu(a)>0$ for $a \notin O_{\mathcal{L}}$.

Condition (Z2) is monotonicity, (Z2)) is strict monotonicity. (Z3) is similar to submodularity with a correction term depending on the difference between $Z_{1} \cap Z_{2}$ and $Z_{1} \wedge Z_{2}$ (the meet is always a subset of the intersection). Some remarks are due.

Remark 1. (Z3) trivially holds when $Z_{1}$ and $Z_{2}$ are comparable, thus it is enough to require it to hold for incomparable $Z_{1}$ and $Z_{2}$.

Remark 2. (Z1) and (Z4) implies $\mu(a)=0$ for $a \in O_{\mathcal{L}}$ as $0 \leq \mu(a) \leq \lambda\left(O_{\mathcal{L}}\right)=$ 0. (Z5) a) trivially follows from $\left(\mathrm{Z}^{*}\right)$.

Remark 3. In conditions (Z2), (Z2*) and (Z3) the subset for which $\mu$ is applied is always disjoint from $O_{\mathcal{L}}$, thus the values $\mu(a)$ for $a \in O_{\mathcal{L}}$ are irrelevant.

Remark 4. Conditions (Z2), (Z2*) and (Z3) are homogeneous in $\lambda$. Thus they hold if and only if they hold for the pointed rank function $\lambda(Z)-\lambda\left(O_{\mathcal{L}}\right)$.

Remark 5. Suppose $\mu(a)=0$ for $a \in O_{\mathcal{L}}$ and $\mu(a)=1$ otherwise, and that $\lambda$ is integer valued. Then (Z2*) implies both (Z4) and (Z5). Consequently "(Z1) and (Z2*) and (Z3) and (Z4) and (Z5)" is equivalent to "(Z1) and (Z2*) and (Z3)", which is the same as the list of axioms in [1, Theorem 3.2], as by Remark 3 in this case the measure $\mu$ of a set in the conditions is the same as its cardinality.

\subsection{Cyclic Flats of Polymatroids}

For each polymatroid $\mathcal{M}$ we define a pair of a ranked lattice and a measure as follows: the ranked lattice is collection of cyclic flats endowed with the polymatroid rank, and the measure is $\mu=\mu_{\mathcal{M}}$ generated by the rank of singletons.

Theorem 4. The pair of the ranked lattice $(\lambda, \mathcal{L})$ and the measure $\mu$ is defined from a polymatroid if and only if they satisfy (Z1), (Z2*), (Z3), (Z4) and (Z5). This polymatroid is uniquely defined, and is integer if and only if $\lambda$ and $\mu$ are integer valued.

By Remark 5, the result in [1] characterizing the lattice of cyclic flats of matroids follows immediately from this theorem. The proof proceeds in two stages. The easy part is Claim 5 , which shows that the lattice and the measure coming from a polymatroid satisfy the conditions. The converse follows from the fact that the convolution $\lambda * \mu$ recovers a polymatroid which defines $\lambda$ and $\mu$. This is proved in Claim 13 using a series of claims and lemmas from Sect. 4 highlighting the role of each condition. The uniqueness follows from Corollary 2: $\lambda$ and $\mu$ together determine the polymatroid rank function. 
Claim 5. Let $\mathcal{L}$ be the lattice of cyclic flats of the polymatroid $\mathcal{M}=(f, M)$. Define $\lambda(Z)=f(Z)$ for $Z \in \mathcal{L}$. Properties (Z1), (Z2*), (Z3), (Z4), (Z5) hold for $(\lambda, \mathcal{L})$ and $\mu=\mu_{\mathcal{M}}$.

Proof. The minimal cyclic flat $O_{\mathcal{L}}$ is the set of loops, i.e., elements with rank zero. This proves (Z1) and (Z5); (Z4) is a simple consequence of monotonicity.

If $Z_{1}<Z_{2}$ then $f\left(Z_{1}\right)<f\left(Z_{2}\right)$ as $Z_{1}$ is a flat and $Z_{2}$ is a proper extension of $Z_{1}$. Suppose by contradiction that $f\left(Z_{2}\right)-f\left(Z_{1}\right) \geq \mu\left(Z_{2}-Z_{1}\right)$, and let $a \in Z_{2}-Z_{1}$. Then $f\left(Z_{1}\right)+\mu\left(Z_{2}-Z_{1} a\right) \geq f\left(Z_{2}-a\right)$ by submodularity, and

$$
f(a)+f\left(Z_{2}-a\right) \geq f\left(Z_{2}\right) \geq f\left(Z_{1}\right)+\mu\left(Z_{2}-Z_{1}\right) \geq f(a)+f\left(Z_{2}-a\right),
$$

which means $f\left(Z_{2}\right)=f\left(Z_{2}-a\right)+f(a)$, thus $Z_{2}$ is not cyclic, proving $\left(Z_{2}^{*}\right)$.

Finally, (Z3) follows from Lemma 1, since $Z_{1} \vee Z_{2}=\operatorname{cl}\left(Z_{1} \cup Z_{2}\right)$, the ranks are equal $f\left(Z_{1} \vee Z_{2}\right)=f\left(Z_{1} \cup Z_{2}\right)$. Let $F=Z_{1} \cap Z_{2}$; it is a flat, and $C=Z_{1} \wedge Z_{2}$ is the maximal cyclic flat contained in $F$. By Lemma 1

$$
f(F)=f\left(Z_{1} \cap Z_{2}\right)=f(C)+\mu_{f}(F-C) .
$$

Combining these with the submodularity

$$
f\left(Z_{1}\right)+f\left(Z_{2}\right) \geq f\left(Z_{1} \cup Z_{2}\right)+f\left(Z_{1} \cap Z_{2}\right)
$$

we get the inequality in (Z3).

\section{Convolution Properties}

In this section, $(\lambda, \mathcal{L})$ is a fixed ranked lattice and $\mu$ is a measure, both defined on subsets of $M$. In this and subsequent Sections the shorter notation $r$ will be used for the convolution $\lambda * \mu$ from Sect. 2.3:

$$
r: A \mapsto \min \{\lambda(Z)+\mu(A-Z): Z \in \mathcal{L}\} .
$$

Theorem 6. If (Z3) holds, then $(r, M)$ is a polymatroid.

Proof. First observe that for arbitrary subsets $A, B, Z_{A}, Z_{B}$ of $M$ we have

$$
\begin{aligned}
& \mu\left(A-Z_{A}\right)+\mu\left(B-Z_{B}\right) \geq \\
& \quad \mu\left(A \cap B-Z_{A} \cap Z_{B}\right)+\mu\left(A \cup B-Z_{A} \cup Z_{B}\right)
\end{aligned}
$$

Indeed, if $i \in A \cap B-Z_{A} \cap Z_{B}$ then $i$ is in both $A$ and $B$ and neither in $Z_{A}$ nor $Z_{B}$, thus it is in either $A-Z_{A}$ or $B-Z_{B}$. If $\left.i \in A \cup B-Z_{A} \cup Z_{B}\right)$, then $i$ is in neither $Z_{A}$ nor $Z_{B}$, thus, again, it is in either $A-Z_{A}$ or $B-Z_{B}$. Finally, if $i$ is in both sets on the right hand side of (3), then $i$ is in both $A$ and $B$, and not in $Z_{A}$ neither in $Z_{B}$, thus $i$ is in both sets on the left hand side.

The convolution $(r, M)$ is a polymatroid if $r$ is non-negative, monotone, and submodular. Non-negativity is clear from the definition (2) as both $\lambda$ and $\mu$ are non-negative. Let $A$ and $B$ be subsets of $M ; r(A)=\lambda\left(Z_{A}\right)+\mu\left(A-Z_{A}\right)$ and $r(B)=\lambda\left(Z_{B}\right)+\mu\left(B-Z_{B}\right)$. If $A \subseteq B$ then

$$
r(A) \leq \lambda\left(Z_{B}\right)+\mu\left(A-Z_{B}\right) \leq \lambda\left(Z_{B}\right)+\mu\left(B-Z_{B}\right)=r(B),
$$


showing monotonicity. The first inequality holds as $r(A)$ is the minimum of $\lambda(Z)+\mu(A-Z)$ as $Z$ runs over the lattice elements. To check submodularity we use $Z_{A} \wedge Z_{B}$ and $Z_{A} \vee Z_{B}$ to estimate $r(A \cap B)$ and $r(A \cup B)$, respectively, as follows:

$$
\begin{aligned}
& r(A \cap B) \leq \lambda\left(Z_{A} \wedge Z_{B}\right)+\mu\left(A \cap B-Z_{A} \wedge Z_{B}\right), \\
& r(A \cup B) \leq \lambda\left(Z_{A} \vee Z_{B}\right)+\mu\left(A \cup B-Z_{A} \vee Z_{B}\right) .
\end{aligned}
$$

Using condition (Z3), the submodularity $r(A)+r(B) \geq r(A \cup B)+r(A \cap B)$ follows if

$$
\begin{array}{r}
\mu\left(A-Z_{A}\right)+\mu\left(B-Z_{B}\right)+\mu\left(Z_{A} \cap Z_{B}-Z_{A} \wedge Z_{B}\right) \\
\quad \geq \mu\left(A \cap B-Z_{A} \wedge Z_{B}\right)+\mu\left(A \cup B-Z_{A} \vee Z_{B}\right) .
\end{array}
$$

As

$$
\mu\left(A \cap B-Z_{A} \wedge Z_{B}\right) \leq \mu\left(A \cap B-Z_{A} \cap Z_{B}\right)+\mu\left(Z_{A} \cap Z_{B}-Z_{A} \wedge Z_{B}\right)
$$

(the right hand side is a disjoint union), and

$$
\mu\left(A \cup B-Z_{A} \vee Z_{B}\right) \leq \mu\left(A \cup B-Z_{A} \cup Z_{B}\right)
$$

as $Z_{A} \vee Z_{B}$ is a subset of $Z_{A} \cup Z_{B}$, (3) gives the required inequality.

Lemma 7. Suppose $a \notin A$ and $A$ is disjoint from $Z \in \mathcal{L}$. If $r(a A Z)=\lambda(Z)+$ $\mu(a A)$, then $r(a A Z)=r(A Z)+\mu(a)$.

Proof. We show that $r(A Z)=\lambda(Z)+\mu(A)$, from here the claim follows. Let $r(A Z)=\lambda\left(Z^{\prime}\right)+\mu\left(A Z-Z^{\prime}\right)$ for some $Z^{\prime} \in \mathcal{L}$. By definition, $r(a A Z)$ is the minimum of $\lambda\left(Z^{\prime \prime}\right)+\mu\left(a A Z-Z^{\prime \prime}\right)$ as $Z^{\prime \prime}$ runs over the lattice elements. In particular,

$$
\lambda(Z)+\mu(a A)=r(a A Z) \leq \lambda\left(Z^{\prime}\right)+\mu\left(a A Z-Z^{\prime}\right) .
$$

Similar consideration applied to $r(A Z)$ gives

$$
\lambda\left(Z^{\prime}\right)+\mu\left(A Z-Z^{\prime}\right)=r(A Z) \leq \lambda(Z)+\mu(A Z-Z)=\lambda(Z)+\mu(A)
$$

as $A Z-Z=A$ by assumption. Combining them we get

$$
\mu(a A)+\mu\left(A Z-Z^{\prime}\right) \leq \mu\left(a A Z-Z^{\prime}\right)+\mu(A),
$$

which holds only if they are equal. Consequently we have equality in (4) as was required.

Lemma 8. (a) (Z1) implies $r(a)=0$ for $a \in O_{\mathcal{L}}$, and $r(a) \leq \mu(a)$ otherwise. (b) (Z4) implies $r(a) \geq \mu(a)$ for all $a \in M$. (c) (Z5) implies $r(a)>0$ for $a \notin O_{\mathcal{L}}$.

Proof. Immediate from the conditions and from the fact that $r(a)$ is the minimum of $\lambda(Z)+\mu(a-Z)$ as $Z$ runs over $\mathcal{L}$.

Lemma 9. (a) Assume (Z2) and (Z3). For every pair of lattice elements $Z, Z^{\prime} \in \mathcal{L}$ we have

$$
\lambda\left(Z \vee Z^{\prime}\right) \leq \lambda\left(Z^{\prime}\right)+\mu\left(Z-Z^{\prime}\right)
$$


(b) Assume (Z2*) and (Z3). For every pair $Z, Z^{\prime}$ in $\mathcal{L}$ such that $Z \not Z Z^{\prime}$ the above inequality is strict.

Proof. If $Z \leq Z^{\prime}$ then $Z \vee Z^{\prime}=Z^{\prime}$, thus the two sides are equal. When $Z^{\prime}<Z$ then the inequality (strict inequality) follows from condition (Z2) (condition $\left(Z 2^{*}\right)$, respectively). Finally, if $Z$ and $Z^{\prime}$ are incomparable, then apply (Z2) (or $\left(\mathrm{Z}^{*}\right)$ ) for $Z \wedge Z^{\prime}$ and $Z^{\prime}$, and (Z3) for $Z$ and $Z^{\prime}$ to get

$$
\begin{aligned}
\lambda(Z) & \leq \lambda\left(Z \wedge Z^{\prime}\right)+\mu\left(Z-Z \wedge Z^{\prime}\right) \\
\lambda\left(Z \wedge Z^{\prime}\right)+\lambda\left(Z \vee Z^{\prime}\right) & \leq \lambda(Z)+\lambda\left(Z^{\prime}\right)-\mu\left(Z \cap Z^{\prime}-Z \wedge Z^{\prime}\right) .
\end{aligned}
$$

Their sum is the claimed inequality. When using $\left(\mathrm{Z} 2^{*}\right)$, the first inequality is strict, thus the sum is strict as well.

Claim 10. Assume (Z2) and (Z3). $r(A)=\lambda(A)$ for every $A \in \mathcal{L}$.

Proof. Using $Z=A$ in the definition $r(A)=\min \{\lambda(Z)+\mu(A-Z)\}$ gives $r(A) \leq \lambda(A)$. To show the converse, condition (Z2) gives $\lambda(A) \leq \lambda(A \vee Z)$, and by Lemma 9 a),

$$
\lambda(A) \leq \lambda(A \vee Z) \leq \lambda(Z)+\mu(A-Z)
$$

for every $Z \in \mathcal{L}$, thus $\lambda(A) \leq r(A)$.

Recall that property (Z3) implies that the convolution is a polymatroid (Theorem 6).

Claim 11. Assume (Z1), (Z3) and (Z5). Every cyclic flat of the convolution is an element of the lattice $\mathcal{L}$.

Proof. (Z3) implies that the convolution $(r, M)$ is a polymatroid. Suppose $F \subseteq M$ is a flat in it, and $r(F)=\lambda(Z)+\mu(F-Z)$ for some $Z \in \mathcal{L}$. Now

$$
r(F Z) \geq r(F)=\lambda(Z)+\mu(F-Z)=\lambda(Z)+\mu(F Z-Z) \geq r(F Z),
$$

consequently $r(F)=r(F Z)$. As $F$ is a flat, $Z \subseteq F$. Suppose $F-Z$ is not empty, let $F-Z=a A$ with $a \notin A$. As $r(a A Z)=\lambda(Z)+\mu(a A)$, Lemma 7 gives $r(F)-r(F-a)=\mu(a)$. By Lemma 8 (a) and (c) $\mu(a) \geq r(a)>0$ (and then $\mu(a)$ and $r(a)$ must be equal), thus $F$ is not cyclic.

Claim 12. Assume (Z2*), (Z3), (Z4), (Z5) (b). Every $Z \in \mathcal{L}$ is a cyclic flat in $(r, M)$.

Proof. By Claim 10, $r(Z)=\lambda(Z)$ for all lattice elements. First we check that $Z \in \mathcal{L}$ is a flat. Let $a \notin Z$, we want to show that $a Z$ has larger rank than $Z$. As $a \notin O_{\mathcal{L}}$, condition (Z5) b) says $\mu(a)>0$. Suppose $r(a Z)=\lambda\left(Z^{\prime}\right)+\mu\left(a Z-Z^{\prime}\right)$. If $Z<Z^{\prime}$ then $\lambda(Z)<\lambda\left(Z^{\prime}\right)$ by $\left(Z 2^{*}\right)$. If $Z=Z^{\prime}$ then $r(a Z)=\lambda(Z)+\mu(a)>$ $\lambda(Z)$. Otherwise $Z$ is not below $Z^{\prime}$, and then Lemma 9 (b) gives

$$
\lambda(Z) \leq \lambda\left(Z \vee Z^{\prime}\right)<\lambda\left(Z^{\prime}\right)+\mu\left(Z-Z^{\prime}\right) \leq r(a Z)
$$

as required. To show that $Z \in \mathcal{L}$ is cyclic, let $a \in Z$ and suppose by contradiction that $r(a)>0$ and $r(Z-a)=r(Z)-r(a)$. Let

$$
r(Z-a)=\lambda\left(Z^{\prime}\right)+\mu\left(Z-a Z^{\prime}\right)=\lambda(Z)-r(a) .
$$


As $\lambda\left(Z^{\prime}\right)<\lambda(Z), Z \leq Z^{\prime}$ is impossible by $\left(Z 2^{*}\right)$. Thus $Z$ is not below $Z^{\prime}$, and Lemma 9 (b) gives

$$
\begin{aligned}
\lambda(Z) \leq \lambda\left(Z \vee Z^{\prime}\right) & <\lambda\left(Z^{\prime}\right)+\mu\left(Z-Z^{\prime}\right) \\
& \leq \lambda\left(Z^{\prime}\right)+\mu\left(Z-a Z^{\prime}\right)+\mu(a) \\
& =\lambda(Z)-r(a)+\mu(a) .
\end{aligned}
$$

However, this is impossible as by Lemma $8(\mathrm{~b}), r(a) \geq \mu(a)$.

Putting together all details, the second part of Theorem 4 follows easily.

Claim 13. Suppose the ranked lattice $(\lambda, \mathcal{L})$ and the measure $\mu$ satisfy conditions (Z1), (Z2*), (Z3), (Z4) and (Z5). The convolution $\lambda * \mu$ recovers a polymatroid $\mathcal{M}$ such that $(\lambda, \mathcal{L})$ is the lattice of cyclic flats of $\mathcal{M}$ endowed with the polymatroid rank, and $\mu=\mu_{\mathcal{M}}$.

Proof. By Theorem 6, the convolution is a polymatroid. $\mu=\mu_{\mathcal{M}}$ follows from Lemma 8 c). By Claims 11 and 12 elements of the lattice $\mathcal{L}$ are precisely the cyclic flats of $\mathcal{M}$. Finally, Claim 10 says that lattice and polymatroid ranks are equal.

\section{An Example}

An illustrative example for using convolution is a proof of Helgason's theorem [6] saying that integer polymatroids are factors of matroids. For other examples see [3]. Let $\mathcal{M}=(f, M)$ be an integer polymatroid. For $i \in M$ find sets $M_{i}$ disjoint from each other such that $M_{i}$ and $M$ intersect in the singleton $\{i\}$ and $M_{i}$ has exactly $\max \{1, f(i)\}$ elements. The lattice $\mathcal{L}$ consists of subsets $Z \subseteq N=\bigcup_{i} M_{i}$ which have the property

$$
\text { if } M_{i} \cap Z \neq \emptyset, \quad \text { then } M_{i} \subseteq Z \text {. }
$$

The meet and join are the union and intersection, and $O_{\mathcal{L}}$ is the empty set. Define the rank $\lambda$ as

$$
\lambda: Z \mapsto f(Z \cap M)
$$

The measure is the expected one: for $a \in M_{i}$ let $\mu(a)=\min \{1, f(i)\}$. It is a routine to check that conditions (Z1), (Z2), (Z3) and (Z4) hold. Let $\mathcal{N}=(r, N)$ be the convolution of the ranked lattice and the measure. It is clearly integer, and by Theorem 6 it is a polymatroid. By Lemma $8 r(a)=\mu(a)$, consequently the rank of singletons is either zero or one, which shows that $\mathcal{N}$ is a matroid. Finally, Claim 10 says $r(Z)=\lambda(Z)$ for all $Z \in \mathcal{L}$, therefore $\mathcal{M}$ is a factor of $\mathcal{N}$ as required.

Helgason's theorem is a special case of a more general statement. In the construction above each singleton $i \in M$ with rank $f(i) \geq 2$ is replaced by the free matroid of rank $f(i)$. Actually any matroid with this rank can be used which is an immediate consequence of Theorem 14. Let us proceed with some definitions. 
The convolution of two ranked lattices $\left(\lambda_{1}, \mathcal{L}_{1}\right)$ and $\left(\lambda_{2}, \mathcal{L}_{2}\right)$ is the function on subsets of $I_{\mathcal{L}_{1}} \cup I_{\mathcal{L}_{2}}$ defined as

$$
A \mapsto \min _{Z_{1}, Z_{2}}\left\{\lambda_{1}\left(Z_{1}\right)+\lambda_{2}\left(Z_{2}\right): A \subseteq Z_{1} \cup Z_{2}\right\}
$$

as $Z_{1}$ runs over elements of $\mathcal{L}_{1}$ and $Z_{2}$ runs over elements of $\mathcal{L}_{2}$. If $\mathcal{L}_{2}$ is the complete subset lattice and $\lambda_{2}$ is a measure, then this formula is equivalent to (1) from Sect. 2.3. In the very special case of Theorem 14 below, the convolution of two ranked lattices defines a polymatroid. To find general and useful conditions implying the same conclusion would be an interesting problem.

Let $P, M$ be disjoint sets, and let $c \notin M \cup P$. Assume $\mathcal{M}=(f, M c)$ and $\mathcal{N}=(g, P)$ are polymatroids with $f(c)=g(P)$. The polymatroid $(r, M \cup P)$ infiltrates $P$ under $c$ if for all subsets $A \subseteq M$ and $B \subseteq P$ we have

$$
\begin{aligned}
r(A) & =f(A), r(B)=g(B), \\
r(A P) & =f(A c),
\end{aligned}
$$

that is, $r$ extends both $f \uparrow M$ and $g$, and inserts $P$ in place of $c$.

Theorem 14. For each $\mathcal{M}=(f, M c)$ and $\mathcal{N}=(g, P)$ with $f(c)=g(P)$ one can infiltrate $P$ under $c$.

Proof. Define two ranked lattices on subsets of $M \cup P$ as follows:

$$
\begin{aligned}
& \mathcal{L}_{1}=\left\{Z_{1} \subseteq M P: Z_{1} \cap P=\emptyset, \text { or } Z_{1} \cap P=P\right\}, \\
& \mathcal{L}_{2}=\left\{Z_{2} \subseteq M P: Z_{2} \cap M=\emptyset\right\}
\end{aligned}
$$

with rank functions

$$
\begin{aligned}
& \lambda_{1}\left(Z_{1}\right)= \begin{cases}f\left(Z_{1} \cap M\right) & \text { if } Z_{1} \cap P=\emptyset, \\
f\left(c\left(Z_{1} \cap M\right)\right) & \text { if } Z_{1} \cap P=P ;\end{cases} \\
& \lambda_{2}\left(Z_{2}\right)=g\left(Z_{2} \cap P\right) .
\end{aligned}
$$

Let the convolution of the ranked lattices be $(r, M P)$. We claim that this is the required extension. As both $\lambda_{1}$ and $\lambda_{2}$ are monotone, the minimum is taken when $Z_{1}$ and $Z_{2}$ is the smallest possible. Consequently for every $A \subseteq M P$,

$$
r(A)=\min \{f(A \cap M)+g(A \cap P), f(c(A \cap M))\} .
$$

Using that $f(c)=g(P)$, conditions in (5) follow easily. Thus one has to check only that $(r, M P)$ is a polymatroid. Non-negativity and monotonicity is clear, and submodularity can be shown by a case by case checking depending on which terms in (6) provide the smaller value.

\section{Acknowledgements}

Open access funding provided by Central European University. The author would like to thank the generous support of the Institute of Information Theory and Automation of the CAS, Prague. The research reported in this paper was supported by GACR project number 19-04579S, and partially by the Lendület program of the HAS. The author would like to thank the reviewers 
for their careful reading and checking. Their numerous invaluable comments and suggestions have been of significant help for improving the presentation.

Open Access. This article is licensed under a Creative Commons Attribution 4.0 International License, which permits use, sharing, adaptation, distribution and reproduction in any medium or format, as long as you give appropriate credit to the original author(s) and the source, provide a link to the Creative Commons licence, and indicate if changes were made. The images or other third party material in this article are included in the article's Creative Commons licence, unless indicated otherwise in a credit line to the material. If material is not included in the article's Creative Commons licence and your intended use is not permitted by statutory regulation or exceeds the permitted use, you will need to obtain permission directly from the copyright holder. To view a copy of this licence, visit http://creativecommons. org/licenses/by/4.0/.

Publisher's Note Springer Nature remains neutral with regard to jurisdictional claims in published maps and institutional affiliations.

\section{References}

[1] J.E. Bonin, A. Mier (2008) The lattice of cyclic flats of a matroid, Annals of Combinatorics, vol 12/2, pp.155-170

[2] L. Csirmaz (2019) One-adhesive polymatroids, arXiv:1904.07565, to appear in Kybernetika

[3] L. Csirmaz (2019) Sticky matroids and convolution, arXiv:1909.02353

[4] J. Edmonds (1970) Submodular functions, matroids, and certain polyhedra, Combinatorial Structures and their Applications (Proc. Calgary Internat. Conf., Calgary, Alta., 1969) Gordon and Breach, New York, pp. 69-87

[5] R. Freij-Hollanti, C. Hollanti, T. Westerbäck (2017) Matroid theory and storage codes: bounds and constructions, In: M. Greferath et al. (eds) Network Coding and Subspace Designs, Springer.

[6] T. Helgason (1974) Aspects of the theory of hypermatroids, In: Berge C., RayChaudhuri D. (eds) Hypergraph Seminar, Lecture Notes in Mathematics, vol 411. Springer, Berlin, Heidelberg

[7] L. Lovász (1982) Submodular functions and convexity. Mathematical Programming - The State of the Art (A. Bachem, M. Grötchel and B. Korte, eds.), Springer-Verlag, Berlin, 234-257.

[8] F. Matúš (2007) Adhesivity of polymatroids, Discrete Mathematics 307 pp. $2464-2477$

[9] F. Matúš, L. Csirmaz (2016) Entropy region and convolution, IEEE Trans. Inf. Theory 62 6007-6018 
[10] J. G. Oxley (2011) Matroid theory, second edition, Oxford University Press, Oxford.

[11] J. A. Sims (1980) Some problems in Matroid Theory, Ph.D. dissertation, Linacre College, Oxford University, Oxford

[12] T. Westerbäck, M. Grezet, R. Freij-Hollanti, C. Hollanti (2018) On the polymatroidal structure of quasi-uniform codes with applications to heterogeneous distributed storage, 23rd International Symposium on Mathematical Theory of Networks and Systems, Hong Kong University of Science and Technology, Hong Kong pp 641-647

Laszlo Csirmaz

Central European University

Budapest

Hungary

e-mail: csirmaz@renyi.hu

Received: 13 October 2019.

Accepted: 29 July 2020. 